\section{PRESCRIÇÃO FARMACÊUTICA DE MEDICAMENTOS FITOTERÁPICOS}

\author{
Paola Alvares Marques'; \\ Marina Maki Moriya; \\ Thainá Aparecida Simão3; \\ Gabriel Dias"; \\ Valéria Maria de Souza Antunes; \\ Carlos de Oliveira Rocha ${ }^{6}$
}

\begin{abstract}
${ }^{1}$ Discente da Universidade Anhembi Morumbi
2Discente da Universidade Anhembi Morumbi

${ }^{3}$ Discente da Universidade Anhembi Morumbi

${ }^{4}$ Discente da Universidade Anhembi Morumbi

${ }^{5}$ Docente da Universidade Anhembi Morumbi.

${ }^{6}$ Docente da Universidade Anhembi Morumbi.
\end{abstract}

Recebido em novembro de 2018.

Aceito em janeiro de 2019.

\section{Resumo}

A prescrição de fitoterápicos por farmacêuticos é uma área recente e ainda em desenvolvimento e, mesmo sendo a terapia medicinal mais antiga do mundo, sempre foi prescrito por médicos. Sabe-se muito pouco sobre suas interações medicamentosas e alimentares e sobre suas toxicidades, já que são compostos de extrema complexidade. Os medicamentos vegetais, que em tempos não muito distantes eram uma forma de medicina empírica, tornaram-se hoje um grande mercado e cada vez mais se investe em pesquisas ao redor do mundo em busca de novas moléculas advindas da natureza. Este trabalho visa analisar a questão da prescrição farmacêutica de fitoterápicos ser mais utilizada pela população, visto que é uma possibilidade acessível e que, aparentemente, é desconhecida pela população, que não está habituada a consultas com o farmacêutico prescritor. Foram estudados 107 prontuários preenchidos durante as consultas realizadas com os alunos de graduação e pós-graduação do curso de Farmácia, acompanhados de um professor farmacêutico prescri- tor de fitoterapia no Centro Integrado de Saúde (CIS), dentro do Campus Centro da Universidade Anhembi Morumbi. Concluímos que apenas 20\% da população atendida retornou para suas consultas de acompanhamento. Os dados obtidos mostram que a população não possui conhecimento sobre esta atribuição do farmacêutico, fato que se fosse exposto, poderia ajudar a população como um todo, uma vez que o farmacêutico faz parte do sistema de saúde e é o profissional detentor do conhecimento sobre interações medicamentosas e toxicológicas, entre outras.

Palavras-chave: Prescrição Farmacêutica. Fitoterapia. Medicamentos.

\section{Descrição e importância da fitoterapia na prática terapêutica}

Os fitoterápicos, sejam eles com finalidades profiláticas, curativas, paliativas ou para diagnósticos, são reconhecidos pela Organização Mundial da Saúde desde $1978^{1}$. Sempre foram um recurso muito valioso e muito procurado pelo homem que buscou na natureza fontes para melhorar a sua qualidade de vida. A evolução do conhecimento sobre os fitoterápicos e suas moléculas fez com que a própria medicina tradicional evoluísse, pois, novas moléculas foram criadas baseando-se nas moléculas oriundas das plantas ${ }^{2}$.

De acordo com Ministério da Saúde, fitoterapia é "uma terapêutica caracterizada pela utilização de plantas medicinais em suas diferentes formas farmacêuticas, sem a utilização de substâncias ativas isoladas, ainda que de origem vegetal, cuja abordagem incentiva o desenvolvimento comunitário, a solidariedade e a participação social" ${ }^{\text {. }}$. Equivocadamente, muitas pessoas chamam a fitoterapia de "terapia alternativa" ou de "medicina de pobre". Essa terapia é um dos métodos mais antigos e ricos já utilizados pela medicina natural. A fitoterapia é muito mais do que preparar um chá, significa a riqueza da cultura milenar, sabedoria, conhecimento acumulado e par- 
tilhado entre gerações. No Brasil, a medicina popular é um reflexo das uniões étnicas de todos os povos que aqui habitavam, como os índios e os imigrantes, que disseminaram seu conhecimento sobre as plantas locais ou trazidas para cá e seus usos, passando esses conhecimentos de geração em geração ${ }^{4,5}$.

Ao longo dos anos, inúmeros foram os avanços científicos na área da fitoterapia. Hoje sabemos que os processos vitais de biossíntese são os responsáveis pela formação, acúmulo e degradação de inúmeras substâncias orgânicas no interior das células que formam os diversos tecidos dos organismos animais e plantas. Das plantas são extraídos diversos ativos e grande parte deles são responsáveis pela aplicabilidade na alimentação e na saúde, o que pode ser um estímulo ao desenvolvimento do conhecimento de muitas plantas dentro do âmbito da química orgânica e suas estruturas, que nós fornece compostos que são extremamente diversificados ${ }^{6}$.

Trata-se da fitoquímica, cuja função é conhecer e identificar os constituintes químicos das plantas e ou o grupo de metabólitos secundários que se tornam relevantes ${ }^{7}$. Já a pesquisa científica com plantas medicinais envolve inúmeros aspectos, o que permite aos pesquisadores conhecimentos amplos e que incluem, desde misticismo de muitas seitas e práticas de saúde que utilizam das plantas medicinais, até o estudo detalhado de uma espécie vegetal ${ }^{8}$. Ao buscar obter substâncias ativas de plantas, o principal aspecto relevante consiste em buscar informações na medicina popular. Hoje em dia, é sabido que é muito mais provável encontrar atividade biológica em plantas de uso na medicina popular, do que em plantas escolhidas ao acaso ${ }^{9}$.

A fitoterapia pode ser considerada parte integral da terapêutica desde o início dos tempos até os dias atuais; sua integração na terapêutica não vem apenas de base histórica, mas também deriva da parte química, radicada na estrutura dos princípios ativos. Grande parte dos fármacos empregados atualmente derivam direta ou indiretamente de princípios ativos que inicialmente foram isolados de plantas. Muitos desses princípios ativos isolados exercem ações farmacológicas potentes e produzem efeitos rápidos ${ }^{10}$. Sendo assim, estudos de plantas e sua aplicação clínica para fins terapêuticos são de fundamental importância para estabelecer diferenças entre os benefícios que a fitoterapia pode oferecer e mitos que foram se desenhando ao longo dos anos.

\section{Uso racional de fitoterápicos}

Segundo Simões ${ }^{7}$, em estudos fotoquímicos que abrangem a utilização de vegetais, é comum observar que existe o pensamento de que plantas medicinais de uso tradicional já foram testadas e aprovadas, acarretando assim o uso inadequado e abusivo, principalmente pela população de baixa renda que acredita na autossugestão e na esperança de cura, não cogitando que as plantas medicinais que podem aliviar sintomas, também podem induzir o paciente a desconsiderar sinais importantes, mascarar sintomas e retardar o atendimento médico, podendo levar então à patologias graves. Corre o risco de consequências às vezes irremediáveis, visto que a adesão para um tratamento com um medicamento fitoterápico está arraigada ao paradigma, "se é natural não faz mal", estabelecido por falta de informação ou pelo conhecimento popular, trazido através do tempo e contribuindo para o uso indiscriminado ${ }^{11}$.

A hipersensibilidade talvez seja um dos efeitos colaterais mais apresentados. Causados, normalmente, pelo uso irracional de plantas medicinais, seus efeitos podem variar de uma dermatite temporária até um choque anafilático ${ }^{12}$. Para a segurança do usuário, 2000 Produtos Fitoterápicos foram classificados pelo Ministério da Saúde como Medicamentos Fitoterápicos e passaram a ter uma legislação específica. A Anvisa passou a fiscalizar sua procedência, eficácia e segurança no uso da classe destes medicamentos ${ }^{13}$. A Política Nacional de Medicina Tradicional e a Regulamentação de Medicamentos Fitoterápicos criada em 2006 discute a respeito das políticas da medicina tradicional fitoterápica. O Brasil inclui-se a esta política porque possui a maior diversidade genética vegetal do mundo, com cerca de 55.000 espécies catalogadas de um total estimado entre 350.000 e 550.000 espécies ${ }^{14}$.

O Sistema Nacional de Informações Tóxico-Farmacológicas (SINITOX) registrou a ocorrência de 8.501 casos de intoxicação por plantas no Brasil no período de 2004 a 2008. Desses registros, 12,4\% estavam relacionadas a circunstâncias intencionais em 
que o usuário buscava propriedades farmacológicas de plantas ${ }^{15}$. A farmacovigilância de plantas medicinais e fitoterápicos têm se tornado uma preocupação emergente. Poderia avaliar os benefícios e riscos dos produtos, assegurando a qualidade, segurança e eficácia compatíveis com seu uso racional ${ }^{16}$. O fato é que os erros de identificação de espécies de plantas e uso off-label da forma tradicional, podem ser perigosos, levando a superdose, inefetividade terapêutica e reações adversas ${ }^{17}$. Além disso, estudar a classe de medicamentos auxilia na contenção de adulterações propositais e não declaradas. Pode auxiliar na identi- ficação de efeitos adversos oriundos de contaminação por agrotóxicos, metais pesados e microrganismos ${ }^{18}$. Muitas vezes, a planta é popularmente considerada terapêuticas, porém, frequentemente, possui propriedades tóxicas desconhecidas pela população.

No caso do Brasil, as maiores classes de medicamentos fitoterápicos utilizados são psicolépticos (sedativos, ansiolíticos e antidepressivos), antivaricosos e anti-hemorroidários, auxiliares digestivos e hepáticos, antiespasmódicos, tônicos, laxantes, descongestionantes, anti gripais e para circulação cerebral ${ }^{19}$.

Tabela 1: Exemplos de fitoterápicos das classes mais utilizadas.

\begin{tabular}{|l|l|}
\hline Classes de medicamentos & \multicolumn{1}{|c|}{ Fitoterápicos } \\
\hline Psicolépticos & $\begin{array}{l}\text { Lantana alba (Erva-cidreira), Citrus aurantifolia (Laranja-amarga), Matrica- } \\
\text { ria recutita (Camomila), Passiflora incarnata (Maracujá). }\end{array}$ \\
\hline Antigripais & Salix alba (Salgueiro) e Allium sativum (Alho). \\
\hline Antiespasmódicos & $\begin{array}{l}\text { Achillea millefolium (Mil folhas), Achyrocline candicans (Macela, ,Marcela e } \\
\text { marcela-do-campo) e Cinnamomum verum (Canela e canela-do-ceilão). }\end{array}$ \\
\hline Anti- hemorroidários & $\begin{array}{l}\text { Hamamelis virginiana (Hamamélis) e Polygonum punctatum (Erva-de-bicho e } \\
\text { pimenteira-dágua). }\end{array}$ \\
\hline $\begin{array}{l}\text { Antidispépticos (auxiliares } \\
\text { digestivos) }\end{array}$ & $\begin{array}{l}\text { Cynara scolymus (Alcachofra), Baccharis trimera (Carqueja e carqueja-amar- } \\
\text { ga) e Casearia sylvestris (Guaçatonga, erva-de-bugre e erva-de-lagarto). }\end{array}$ \\
\hline
\end{tabular}

Fonte: os autores.

Estima-se que o mercado econômico dos fitoterápicos movimenta cerca de 22 bilhões de dólares por ano. O mercado nacional é composto por 119 empresas que possuem registro junto à ANVISA. Este mercado registrado movimenta, aproximadamente, 1,8 milhões de reais ao ano ${ }^{20}$.

\section{Prescrição de fitoterápicos e profissionais atuantes}

Trabalhar com plantas medicinais é um trabalho multiprofissional. Abrange as mais variadas áreas do conhecimento, desde os aspectos botânicos aos fitoquímicos. Para que todo esse trabalho seja bem executado, são necessários especialistas para cada uma das etapas. Desde o plantio a qualidade do solo, semente, mudas e água para irrigação, até o medica- mento fitoterápico em si, distribuídos nas farmácias. Somente com trabalho e pesquisas é que este mercado se ampliará de forma correta, segura e confiável.

Recomenda-se que medicações extraídas de plantas medicinais devam ser prescritas por profissionais de saúde. No entanto, o desconhecimento destes profissionais sobre plantas medicinais, suas interações com medicamentos alopáticos e sua toxicidade tornam-se fatores preocupantes quando se fala de automedicação dos pacientes ${ }^{21}$. De acordo com o Relatório do Seminário Internacional das Práticas Integrativas e Complementares em Saúde, além de médicos e farmacêuticos, outros profissionais da área de saúde podem prescrever Fitoterápicos ${ }^{22}$. Os resultados mostram o crescimento do uso de métodos alternativos pelos enfermeiros ${ }^{23}$. Em contrapartida, o profissional que menos prescreve fitoterápicos é o odontólogo ${ }^{24}$. 
Gráfico 1: Profissionais que utilizam fitoterapia.

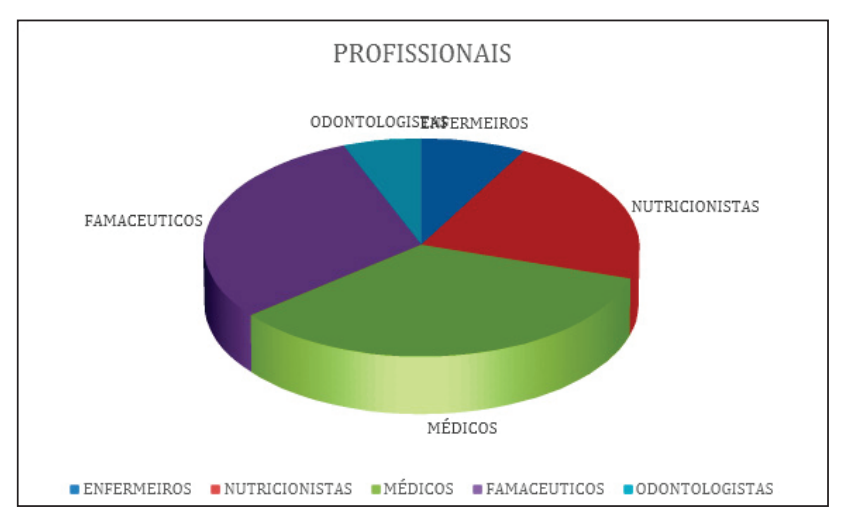

Fonte: Ministério da Saúde, 2015. ${ }^{25}$

Os profissionais sentem-se inseguros no uso da fitoterapia mesmo com o reconhecimento pelos Conselhos de Medicina, Enfermagem e Farmácia ${ }^{26}$. Com exceção dos profissionais farmacêuticos, os outros profissionais citados não tiveram formação durante a graduação sobre o tema fitoterapia, esclarecendo assim, a falta de conhecimentos científicos e práticos ${ }^{24}$. Desta forma, os Conselhos Regionais de Medicina, Enfermagem e Farmácia, aceitam como prescritores somente profissionais com pós-graduação na área. Os farmacêuticos, mesmo com a disciplina em sua grade curricular, devem fazer a pós-graduação e, somente depois, iniciar as atividades na prescrição.

Importância do farmacêutico na fitoterapia

O farmacêutico é o elo entre o conhecimento popular e a ciência, prestando assistência e passando as informações sobre o uso racional de medicamentos, sobre as interações entre medicamentos, fitoterápicos e alimentos. É papel deste profissional apresentar seu conhecimento sobre as plantas, drogas vegetais e drogas ${ }^{27}$. A utilização desta prática é parte essencial no trabalho do farmacêutico, já que a Organização Mundial da Saúde ressalta que $80 \%$ da população mundial necessita das práticas tradicionais no que se refere à atenção primária à saúde e que $67 \%$ das espécies vegetais medicinais do mundo são procedentes de países em desenvolvimento ${ }^{28}$. Com isso, as plantas medicinais se tornaram importantes características da Assistência Farmacêutica e, como suporte para tal atividade, a Agência Nacional de Vigilância Sanitária (ANVISA) publicou a primeira edição de um Formulário de Fitoterápicos da Farmacopeia Brasileira em
2011. Este documento oficializa e padroniza informações. Possui 47 monografias de vegetais para infusos e decoctos, 17 tinturas, um xarope, cinco géis, cinco pomadas, um sabonete, dois cremes, quatro bases farmacêuticas e uma solução conservante. Estão registradas informações sobre a forma correta de preparo e suas indicações, além de restrições de uso de cada espécie, tendo como requisito a qualidade definida nas normas específicas para farmácia de manipulação e farmácias vivas. Conjuntamente foram feitos estudos científicos de todas as formulações incluídas no Formulário e a utilização das mesmas nos serviços de fitoterapia no país. Este Formulário contribui de forma significativa para ampliar a atuação farmacêutica na prescrição de fitoterápicos, pois o documento dá certeza e confiabilidade nas informações prestadas ${ }^{29}$.

Para regulamentar as atribuições farmacêuticas foram criadas as RDC'S $\mathrm{n}^{\circ} 585$, de 29 de agosto de 2013 e no 586, de 29 de agosto de 2013 pelo Conselho Federal de Farmácia (CFF) ${ }^{30,31}$, sendo que a RDC $n^{\circ} 586 / 13$ fala sobre as especificações para a prescrição. Os medicamentos fitoterápicos prescritos por farmacêutico são limitados, uma vez que alguns somente podem ser prescritos por médicos. Essas medidas foram tomadas para que, além da valorização do profissional, a população obtenha a prescrição farmacêutica de medicamentos fitoterápicos como primeira opção, com prescritor habilitado e que saberá ajudá-la nas questões de performance da planta medicinal e da melhor forma de utilizá-la. Embora os medicamentos fitoterápicos abaixo (Tabela 2) sejam isentos de prescrição médica, não significa que seja livres de orientação, o que comprova a importância do conhecimento do profissional farmacêutico.

Para que os cursos de Farmácia seguissem um padrão brasileiro quanto ao ensino, foram criadas as Diretrizes Curriculares Nacionais (DCNs) de Farmácia. A Resolução em vigor é a de no 06 de 2017 que revogou a $\mathrm{n}^{\circ} 02$ de 2002. Ambas contemplam as competências e habilidades que cabem ao profissional farmacêutico terem durante a graduação e depois dela, mas as afirmações feita na resolução anterior vetam qualquer adjetivação que possa dar a conotação de habilitações específicas para o curso, o que muda o cenário do profissional ${ }^{33,34}$. Desta forma, a legislação torna o farmacêutico um profissional sem habilita- 
ção específica para que possa para atuar em todas as frentes farmacêuticas. $\mathrm{O}$ ensino se divide em porcentagens de carga para cada área de conhecimento e não aprimora o profissional em nenhuma. A Resolução em vigor é a de no 06 de 2017 que, no entanto, mo- difica a condição do curso de Farmácia para que este forme profissionais da saúde que possam interagir com outros profissionais da saúde em prol da saúde pública.

Tabela 2: Medicamentos e produtos fitoterápicos isentos de prescrição médica com indicações e respectivas restrições de uso.

\begin{tabular}{|c|c|c|}
\hline $\begin{array}{l}\text { Medicamentos e produtos } \\
\text { fitoterápicos }\end{array}$ & Indicações & Restrições \\
\hline $\begin{array}{l}\text { Aesculus hippocastanum (Casta- } \\
\text { nha da índia) }\end{array}$ & Insuficiência venosa. & - \\
\hline Allium sativum (Alho) & $\begin{array}{l}\text { Hiperlipidemia e hipertensão } \\
\text { arterial leve a moderada. }\end{array}$ & - \\
\hline $\begin{array}{l}\text { Centella asiatica (Centela/ Cen- } \\
\text { tela-asiática) }\end{array}$ & $\begin{array}{l}\text { Insuficiência venosa dos } \\
\text { membros inferiores. }\end{array}$ & - \\
\hline Cynara scolymus (Alcachofra) & $\begin{array}{l}\text { Antidispéptico, antiflatulento, } \\
\text { diurético. }\end{array}$ & - \\
\hline Glycine max (Soja) & Sintomas do climatério. & - \\
\hline Glycyrrhiza glabra (Alcaçuz) & Úlceras gástricas e duodenais. & $\begin{array}{l}\text { Não recomendado uso contínuo por } \\
\text { mais de seis semanas, sem acompanha- } \\
\text { mento médico. }\end{array}$ \\
\hline $\begin{array}{l}\text { Mentha } x \text { piperita (Hortelã-pi- } \\
\text { menta) }\end{array}$ & $\begin{array}{l}\text { Expectorante, carminativo e } \\
\text { antiespasmódico e síndrome } \\
\text { do cólon irritável. }\end{array}$ & $\begin{array}{l}\text { Venda livre para ação expectorante, } \\
\text { carminativo e antiespasmódico. }\end{array}$ \\
\hline Panax ginseng (Ginseng) & $\begin{array}{l}\text { Estado de fadiga física e men- } \\
\text { tal, adaptógeno. }\end{array}$ & Utilizar por no máximo três meses. \\
\hline Paullinia cupana (Guaraná) & Psicoestimulante e astenia. & - \\
\hline $\begin{array}{l}\text { Pimpinella anisum (Erva-doce/ } \\
\text { Anis) }\end{array}$ & $\begin{array}{l}\text { Expectorante, antiespasmódi- } \\
\text { co, carminativo e dispepsias } \\
\text { funcionais. }\end{array}$ & - \\
\hline Plantago ovata (Plantago) & $\begin{array}{l}\text { Obstipação intestinal e sín- } \\
\text { drome do cólon irritável }\end{array}$ & $\begin{array}{l}\text { Venda livre como coadjuvante nos } \\
\text { casos de obstipação intestinal. }\end{array}$ \\
\hline Polygala senega (Polígala) & Bronquite crônica, faringite. & - \\
\hline $\begin{array}{l}\text { Frangula purshiana (Cáscara } \\
\text { Sagrada) }\end{array}$ & Constipação ocasional. & $\begin{array}{l}\text { Não recomendado uso contínuo por } \\
\text { mais de uma semana. }\end{array}$ \\
\hline Salix alba (Salgueiro Branco) & $\begin{array}{l}\text { Antitérmico, anti-inflamató- } \\
\text { rio e analgésico. }\end{array}$ & - \\
\hline
\end{tabular}




\begin{tabular}{|c|c|c|}
\hline Senna alexandrina (Sene) & Laxativo. & - \\
\hline Vaccinium myrtillus (Mirtilo) & $\begin{array}{l}\text { Insuficiência venosa perifé- } \\
\text { rica. }\end{array}$ & - \\
\hline $\begin{array}{l}\text { Zingiber officinale Roscoe (Gen- } \\
\text { gibre) }\end{array}$ & $\begin{array}{l}\text { Náuseas causadas por movi- } \\
\text { mento (cinetose) e pós-cirúr- } \\
\text { gicas. }\end{array}$ & - \\
\hline Arnica montana (Arnica) & $\begin{array}{l}\text { Equimoses, hematomas e } \\
\text { contusões. }\end{array}$ & Não usar em ferimentos abertos. \\
\hline $\begin{array}{l}\text { Calendula officinalis (Calêndu- } \\
\text { la) }\end{array}$ & $\begin{array}{l}\text { Cicatrizante, anti-inflamató- } \\
\text { rio. }\end{array}$ & - \\
\hline Eucalyptus globulus (Eucalipto) & $\begin{array}{l}\text { Antisséptico das vias aéreas } \\
\text { superiores e expectorante. }\end{array}$ & - \\
\hline $\begin{array}{l}\text { Hamamelis virginiana (Ha- } \\
\text { mamélis) }\end{array}$ & $\begin{array}{l}\text { Antihemorroidal e equimo- } \\
\text { ses. }\end{array}$ & - \\
\hline $\begin{array}{l}\text { Harpagophytum procumbens } \\
\text { (Garra do diabo) }\end{array}$ & $\begin{array}{l}\text { Dores articulares moderadas } \\
\text { e dor lombar baixa aguda. }\end{array}$ & - \\
\hline Matricaria recutita (Camomila) & $\begin{array}{l}\text { Antiespasmódico intestinal, } \\
\text { dispepsias funcionais. }\end{array}$ & - \\
\hline $\begin{array}{l}\text { Maytenus ilicifolia (Espinheira- } \\
\text { santa) }\end{array}$ & $\begin{array}{l}\text { Dispepsias, gastrite e úlcera } \\
\text { gastroduodenal. }\end{array}$ & - \\
\hline $\begin{array}{l}\text { Melissa officinalis (Melissa/ } \\
\text { Erva Cidreira) }\end{array}$ & $\begin{array}{l}\text { Carminativo, antiespasmódi- } \\
\text { co e ansiolítico leve. }\end{array}$ & - \\
\hline Mikania glomerata (Guaco) & $\begin{array}{l}\text { Expectorante e broncodilata- } \\
\text { dor. }\end{array}$ & - \\
\hline Passiflora edulis (Maracujá) & Ansiolítico leve. & \\
\hline $\begin{array}{l}\text { Peumus boldus Molina (Boldo/ } \\
\text { Boldo-do-Chile) }\end{array}$ & $\begin{array}{l}\text { Dispepsias funcionais e } \\
\text { distúrbios gastrointestinais } \\
\text { espásticos. }\end{array}$ & - \\
\hline Sambucus nigra (Sabugueiro) & Mucolítico/expectorante. & - \\
\hline $\begin{array}{l}\text { Silybum marianum (Cardo } \\
\text { mariano) }\end{array}$ & Hepatoprotetores & - \\
\hline Symphytum officinale (Confrei) & $\begin{array}{l}\text { Cicatrizante, equimoses, he- } \\
\text { matomas e contusões. }\end{array}$ & $\begin{array}{l}\text { Utilização por no máximo } 4 \text { a } 6 \text { sema- } \\
\text { nas/ano. Não utilizar em lesões abertas. }\end{array}$ \\
\hline $\begin{array}{l}\text { Uncaria tomentosa (Unha de } \\
\text { gato) }\end{array}$ & Anti-inflamatório. & $\begin{array}{l}\text { Não recomendado para gestantes, lac- } \\
\text { tantes e lactentes. }\end{array}$ \\
\hline
\end{tabular}

Fonte: ANVISA, $2014^{(32)}$ 


\section{Trabalho de Atenção Farmacêutica e Prescrição de Fitoterápicos no Centro Integrado de Saúde da Universidade Anhembi Morumbi}

A instituição de ensino Universidade Anhembi Morumbi (UAM) faz parte da Rede Laureate de Universidades, tem experiência de 15 anos no ensino farmacêutico com reconhecimento no MEC $^{35}$. Em 2017 foi desenvolvido um projeto de atenção farmacêutica para os alunos de graduação do curso de Farmácia no Centro Integrado de Saúde (CIS). Este centro, inaugurado em 2010, localiza-se no Campus Mooca-Centro, dispõe de 47 consultórios de atendimento, dois ginásios de fisioterapia e uma piscina ${ }^{36}$. De acordo com o portal da UAM o CIS foi considerado um dos mais amplos e completos centros de atendimento clínico da cidade de São Paulo, por oferecer atendimento, com valores diferenciados ou até mesmo gratuitos, tanto para alunos e colaboradores quanto para a comunidade da região, além de oferecer consultas em dez áreas diferentes, sendo elas Educação Física, Enfermagem, Farmácia, Fisioterapia, Medicina, Naturologia, Nutrição, Podologia, Psicologia e Quiropraxia ${ }^{37}$.

O projeto aplicou-se também aos alunos de pósgraduação em Farmácia Clínica, Prescrição Farmacêutica em Fitoterapia e Produtos Naturais. Realiza-se uma anamnese que, segundo Santos, Veiga e Andra$\mathrm{de}^{38}$ é a primeira fase de um processo, na qual a coleta de dados permite ao profissional de saúde identificar problemas, determinar diagnósticos, planejar e implementar a sua assistência. Neste documento, são coletados dados básicos como idade, peso e altura além de informações para o tratamento farmacoterapêutico. Registrado os dados no prontuário do paciente, os alunos são orientados a pesquisar qual o tratamento recomendado, de acordo com a necessidade individual para cada tratamento. Foram analisados Problemas Relacionados a Medicamentos (PRM's), dificuldades com o tratamento, meios de otimizar o tratamento com indicações não medicamentosas e o uso de medicinas alternativas e fitoterapia. Todo esse estudo é acompanhado pelo professor orientador do projeto, dando assim um prazo de retorno ao paciente para que ele receba as recomendações necessárias.

Tivemos acesso aos prontuários dos pacientes que participaram do projeto com alunos da graduação a partir março de 2017 até junho de 2018. Foram realizados 77 atendimentos, dentre eles haviam $51 \mathrm{mu}-$ lheres e 26 homens, com idades variando de 20 a 76 anos, em sua maioria, idosos encaminhados de fisioterapia. Os dados da pós-graduação contemplam os atendimentos feitos entre as datas de março de 2017 até junho de 2017. Foram atendidos 30 pacientes que buscaram diretamente o serviço de Atenção Farmacêutica, em sua maioria, mulheres em média 60 anos, com queixas de estresse e já vinham com o diagnóstico de hipertensão.

As taxas de retorno para o acompanhamento do tratamento foram de $13 \%$ na graduação e $40 \%$ na pós-graduação. Na somatória dos atendimentos, obtivemos o total de $20 \%$. Esse número mostra a desinformação da possibilidade do atendimento e é um reflexo da desinformação da população em relação ao papel do farmacêutico como um auxiliar no tratamento farmacológico.

Com o intuito da mudança deste conceito, em 2014 foi estabelecido a Lei $\mathrm{n}^{\circ} 13.021$ onde farmácia se torna estabelecimento de saúde e o farmacêutico começa a ser reconhecido como um profissional de saúde ${ }^{39}$. O ministério da Educação estabeleceu nas DCNs de Farmácia de 2017 que o eixo de cuidados em saúde devem ser $50 \%$ da carga horária total durante todo o curso, este eixo engloba a realização de atividades de promoção, proteção e recuperação da saúde, além da prevenção de doenças, e que possibilite às pessoas viverem melhor ${ }^{34}$.

\section{Conclusão}

A fitoterapia é uma área muito vasta, rica, onde as pesquisas são escassas, apesar do grande número de adeptos, consumidores que fazem uso da tradicionalidade medicamentosa, sem os questionamentos científicos necessários sobre toxicidade, interações medicamentosas e alimentares. Para isto se faz necessário o apoio complementar das pesquisas, uma vez que nossas riquezas ambientais na Amazônia são pouco conhecidas pelos pesquisadores nacionais. A Farmacognosia, ciência que estuda parte da farmacologia que trata das drogas ou substâncias medicinais em seu estado natural, antes de serem manipuladas é 
uma disciplina exclusiva do curso de Farmácia e poderia gerar um campo amplo de cuidados farmacêuticos e prescrições farmacêuticas, que incluiriam os cuidados dermatológicos, dentre vários outros. Caberia aos conselhos de classe e ao governo a divulgação desta atividade profissional.

A população desconhece o papel do farmacêutico enquanto prescritor e seus serviços como um profissional apto a atenção ao paciente, para situações onde não haja necessidade de cuidados médicos. Os conselhos Federal e Regionais de Farmácia têm um papel importante em mudar esse cenário, disseminando informações quanto ao trabalho farmacêutico e o quanto ele é benéfico, além da criação de resoluções para regulamentação e ampliação das atividades profissionais. Cabe, também, ao Ministério da Saúde a divulgação através de campanhas para que o profissional Farmacêutico possa desenvolver seu atendimento à população, muitas vezes desafogando os pronto-socorros com cuidados básicos. As universidades devem preparar seus alunos para este tipo de atendimento, qualificando-os para realizar na prática o que é visto em sala de aula, através dos atendimentos monitorados por professores especialistas. Desta forma, com mais consciência e aptidão, o profissional farmacêutico poderá se tornar, efetivamente, o prestador de serviços que procura promover, recuperar e proteger a saúde de um indivíduo ou de uma comunidade.

\section{Referências}

1. Organização Mundial de Saúde (OMS). Cuidados primários de saúde. Brasília, 1978.

2. Organização das nações unidas para a educação, a ciência e a cultura (UNESCO). Culture and Health: Orientation Texts: World Decade for Cultural Development 1988-1997, Document CLT/DEC/PRO. Paris, 1996.

3. Ministério da Saúde. Portaria no. 971, de 03 de maio de 2006. Aprova a Política Nacional de Práticas Integrativas e Complementares (PNPIC) no Sistema Único de Saúde. D.O.U. Diário Oficial da União, Poder Executivo, Brasília, 04 mai. 2006.

4. Lorenzi H, Matos FJ. Plantas medicinais no Brasil: nativas e exóticas. 1 ed. Nova Odessa: Institu- to Plantarum de Estudos da Flora, 2002.

5. Lorenzi $\mathrm{H}$, Matos FJ. Plantas medicinais do Brasil: nativas e exóticas. 2. ed. Nova Odessa: Instituto Plantarum de Estudos da Flora, 2008.

6. Santos EM. Florística etnobotânica e tipagem fitoquímica de espécies medicinais de uso popular nos cerrados dos municípios de Caxias e Timon, Maranhão. Seminário de Iniciação Científica da UEMA 2002. 7. Simões, CMO. Farmacognosia: da planta ao medicamento. 3 eds. Porto Alegre: Ed. da UFSC, 2001.

8. Di stasi LC. Plantas medicinais: arte e ciência - Um guia de estudo interdisciplinar. São Paulo: UNESP, 1996.

9. Yunes RA, Calixto JB. Plantas medicinais sob a ótica da moderna química medicinal. Chapecó: Argos, 2001.

10. Vanaclocha BV, Folcará SC. Fitoterapia: vademécum de prescripción. 4. ed. Barcelona: Masson, 2003.

11. Rossato MB, Budó MLD, Alvim NAT, Zanetti GD, Heisler EV. Saberes e práticas populares de cuidado em saúde com o uso de plantas medicinais. Texto Contexto Enferm, 2012 Abr-Jun; 21(2): 363370.

12. Cunha AP. Plantas e produtos vegetais em cosmética e dermatologia. Lisboa: Fundação Calouste Gulbenkian, 2004. 310 p.

13. Alonso, JR. Fitomedicina: curso para profissionais da área da saúde. São Paulo: Pharmabooks, 2008.

14. Ministério da Saúde (Brasil). Política nacional de plantas medicinais e fitoterápicos. Brasília: Ministério da Saúde, 2006.

15. Sistema Nacional de Informações Tóxico-Farmacológicas. Instituto de comunicação e informação científica e tecnológica em saúde, Fundação Oswaldo Cruz. Estatística anual de casos de intoxicação e envenenamento. Disponível em: <www.sinitox.icict. fiocruz.br>.

16. Silveira PF, Bandeira MAM, Arrais P.S.D. Farmacovigilância e reações adversas às plantas medicinais e fitoterápicos: uma realidade. Rev. Bras. Farmacogn. João Pessoa, 2008.

17. World Health Organization. The importance of pharmacovigilance safety monitoring of medici- 
nal products. Geneva. 2002.

18. World Health Organization. Guidelines on safety monitoring of herbal medicines in pharmacovigilance systems. Geneva. 2004.

19. Valenze FH, Brenzan MA. Perfil de utilização de medicamentos fitoterápicos pela população do município de Boa Esperança PR. Rev. SaBios, 2011;6(1): 17-24.

20. Zuanazzi JAS, Mayorga P. Fitoprodutos e desenvolvimento econômico. Quím. Nova 2010;33(6): 1421-1428.

21. Silva MAB, Melo LVL, Ribeiro RV, Souza JPM, Lima JCS, Martins DTO et al. Levantamento etnobotânico de plantas utilizadas como anti-hiperlipidêmicas e anorexígenas pela população de Nova Xavantina -MT, Brasil. Rev. Bras. Farmacogn, 2010.

22. Brasil. Ministério da Saúde. Secretaria de Atenção à Saúde. Departamento de Atenção Básica. Relatório do $1^{\circ}$ seminário internacional de práticas integrativas e complementares em saúde - PNPIC / Ministério da Saúde, Secretaria de Atenção à Saúde, Departamento de Atenção Básica. - Brasília : Ministério da Saúde, 2009. 196 p.

23. Alves AR, Silva MJP. O uso da fitoterapia no cuidado de crianças com até cinco anos em área central e periférica da cidade de São Paulo. Rev. Esc. Enferm. USP 2003; 37(4):85-91.

24. Menezes VA, Anjos AGP, Pereira MR, Leite AF, Granville-Garcia AF. Terapêutica com plantas medicinais: percepção de profissionais da estratégia de saúde da família de um município do agreste pernambucano. Odonto 2012; 20(39): 111-122.

25. Brasil. Ministério da Saúde. Secretaria de Atenção à Saúde. Departamento de Atenção Básica. Práticas integrativas e complementares: plantas medicinais e fitoterapia na Atenção Básica/Ministério da Saúde. Secretaria de Atenção à Saúde. Departamento de Atenção Básica. Brasília : Ministério da Saúde, 2015.

26. Machado DC, Czermainski SBC, Lopes EC. Percepções de coordenadores de unidades de saúde sobre a fitoterapia e outras práticas integrativas e complementares. Saúde em Debate 2012 outdez;36(95):615-623.

27. Panizza ST. Como prescrever ou recomendar plantas medicinais e fitoterápicos. São Luiz: Conbra- fito, 2010.2

8. Alonso, JR. Tratado de fitomedicina: bases clínicas y farmacológicas. Buenos Aires: Isis, 1998.

29. Agência Nacional de Vigilância Sanitária (Brasil). Formulário de Fitoterápicos da Farmacopéia Brasileira / Agência Nacional de Vigilância Sanitária. Brasília: Anvisa, 2011. 126p.

30. Conselho Federal de Farmácia (Brasil). Resolução RDC no 585 de 29 de agosto de 2013. Dispõe sobre as atribuições clínicas do farmacêutico, 2013.

31. Conselho Federal de Farmácia (Brasil). Resolução RDC no 586 de 29 de agosto de 2013. Regulamenta a prescrição farmacêutica, 2013.

32. Agência Nacional de Vigilância Sanitária (Brasil) Instrução Normativa $n^{\circ} 02$, de 13 de maio de 2014. Publica a "Lista de medicamentos fitoterápicos de registro simplificado" e a "Lista de produtos tradicionais fitoterápicos de registro simplificado", 2014.

33. Conselho Nacional de Educação. Resolução CNE/CES 2/2002. Diário Oficial da União, Brasília, 04 de março de 2002. Seção 1, p. 9.

34. Ministério da Educação (Brasil). Resolução CNE/CES 6/2017. Diário Oficial da União, Brasília, 20 de outubro de 2017, Seção 1, p. 30.

35. Portal Anhembi Morumbi. Farmácia: sobre o curso.

36. Portal Anhembi Morumbi. Centro Integrado de Saúde (CIS).

37. Portal Anhembi Morumbi. Centro Integrado de Saúde (CIS).

38. Santos N, Veiga P, Andrade R. Importância da anamnese e do exame físico para o cuidado do enfermeiro.Rev Bras Enferm, 2011 Mar-Abr; 64(2): 355-358.

39. Brasil. Lei $n^{\circ} 13.021$, de 08 de agosto de 2014. Dispõe sobre o exercício e a fiscalização das atividades farmacêuticas. Diário Oficial da União $11 \mathrm{de}$ agosto de 2014. 